\title{
Metric dimension of some distance-regular graphs
}

\author{
Jun Guo ${ }^{\mathrm{a}}$, Kaishun Wang ${ }^{\mathrm{b}, *}$, Fenggao Li ${ }^{\mathrm{c}}$ \\ ${ }^{a}$ Math. and Inf. College, Langfang Teachers' College, Langfang 065000, China \\ ${ }^{b}$ Sch. Math. Sci. E Lab. Math. Com. Sys., Beijing Normal University, Beijing 100875, China \\ ${ }^{c}$ Dept. of Math., Hunan Institute of Science and Technology, Yueyang 414006, China
}

\begin{abstract}
A resolving set of a graph is a set of vertices with the property that the list of distances from any vertex to those in the set uniquely identifies that vertex. In this paper, we construct a resolving set of Johnson graphs, doubled Odd graphs, doubled Grassmann graphs and twisted Grassmann graphs, respectively, and obtain the upper bounds on the metric dimension of these graphs.
\end{abstract}

Keywords: Metric dimension, Johnson graph, doubled Odd graph, doubled Grassmann graph, twisted Grassmann graph

2010 MSC: 05C12, 05E30

\section{Introduction}

Let $\Gamma$ be a connected graph. For any two vertices $u$ and $v, d(u, v)$ denotes the distance between $u$ and $v$. A resolving set of $\Gamma$ is a set of vertices $S=\left\{v_{1}, \ldots, v_{k}\right\}$ such that, for each vertex $w$, the ordered list of distances $D(w \mid S)=\left(d\left(w, v_{1}\right), \ldots, d\left(w, v_{k}\right)\right)$ uniquely determines $w$. That is, $S$ is a resolving set of $\Gamma$ if, for any two distinct vertices $u$ and $v, D(u \mid S) \neq D(v \mid S)$. The metric dimension of $\Gamma$, denoted by $\mu(\Gamma)$, is the smallest size of all resolving sets of $\Gamma$.

Metric dimension is a well-known parameter in graph theory. It was first introduced in the 1970s, independently by Harary and Melter [12] and by Slater [13]. Computing the metric dimension of a graph is an NP-hard problem [4]. In recent years, a considerable literature has developed in graph theory. Cáceres et al. [7] studied the metric dimension of Cartesian products of graphs. Chartrand et al. [8] determined all connected graphs of order $n$ having metric dimension $1, n-2$ or $n-1$, and presented a new proof on the metric dimension of a tree. An interesting case is that of distance-regular graphs. Bailey et al. [2] obtained an upper bound on the metric dimension of Johnson graphs. Bailey and Meagher [3] constructed a resolving set of Grassmann graphs. Chvátal [9] obtained an upper bound on the metric dimension of Hamming graphs. Feng and Wang [11] obtained an upper bound on the metric dimension of bilinear forms graphs.

In this paper, we construct a resolving set of Johnson graphs, doubled Odd graphs, doubled Grassmann graphs and twisted Grassmann graphs, respectively, and obtain the upper bounds on the metric dimension of these graphs.

\section{Johnson graphs}

For any positive integer $m$, let $[m]=\{1,2, \ldots, m\}$ and let $\left(\begin{array}{c}{[m]} \\ i\end{array}\right)$ be the set of all $i$-subsets of $[m]$. Given integers $2 \leq 2 e \leq n$, the Johnson graph $J(n, e)$ has the vertex set $\left(\begin{array}{c}{[n]} \\ e\end{array}\right)$ such that two vertices $P$ and $Q$ are adjacent if and only if $|P \cap Q|=e-1$. Then $J(n, e)$ is a distance-regular graph with diameter $e$ (see [6]). For any two vertices $P$ and $Q$, we have $d(P, Q)=i$ if and only if $|P \cap Q|=e-i$. For $e=1, J(n, e)$ is a complete graph. It is obvious that $\mu(J(n, 1))=n-1$. For $e=2$, by [4, Corollary 3.33], $\mu(J(3,2))=2, \mu(J(4,2))=3, \mu(J(5,2))=3$ and $\mu(J(n, 2))=\frac{2}{3}(n-i)+i$, where $n \geq 6$ and $n \equiv i(\bmod 3), i \in\{0,1,2\}$. For $e \geq 3$, Bailey et al. obtained the following result.

\footnotetext{
${ }^{*}$ Corresponding author

Email addresses: guojun_lf@163.com (Jun Guo), wangks@bnu.edu.cn (Kaishun Wang), fenggaol@163.com (Fenggao Li)
} 
Proposition 2.1. ([2]) Let $e \geq 3$. Then $\mu(J(n, e)) \leq(e+1)\lceil n /(e+1)\rceil$.

For $n=2 e+1$, we improve the bound of Proposition 2.1 and obtain the following result.

Theorem 2.2. Let $e \geq 3$. Then $\mu(J(2 e+1, e)) \leq 2 e$.

Proof. Let $X=[e]$ and $Y=[2 e] \backslash X$. To prove

$$
\mathcal{M}=\left\{M_{1} \cup\{2 e+1\} \mid M_{1} \in\left(\begin{array}{c}
X \\
e-1
\end{array}\right)\right\} \cup\left\{M_{2} \cup\{2 e+1\} \mid M_{2} \in\left(\begin{array}{c}
Y \\
e-1
\end{array}\right)\right\}
$$

is a resolving set, it is sufficient to show that, for any two distinct vertices $P$ and $Q$, there exists a vertex $W \in \mathcal{M}$ such that $|P \cap W| \neq|Q \cap W|$.

Case 1: $P \cap[2 e] \neq Q \cap[2 e]$. Then there exists a $Z \in\{X, Y\}$ such that $P \cap Z \neq Q \cap Z$. Suppose $s=|P \cap Z| \leq|Q \cap Z|=r$. Then $1 \leq r \leq e$.

Case 1.1: $r=e$. Then $Q=Z$. Since $P \cap Z \neq Q \cap Z, s<e$. If $s=0$, take an $(e-1)$-subset $W_{1}$ of $Z$. Then $W=W_{1} \cup\{2 e+1\} \in \mathcal{M}$ and $|P \cap W| \leq 1<e-1=|Q \cap W|$. If $1 \leq s \leq e-2$, let $W_{1}$ be an $(e-1)$-subset of $Z$ satisfying $\left|P \cap W_{1}\right|=s-1$. Then $W=W_{1} \cup\{2 e+1\} \in \mathcal{M}$ and $|P \cap W| \leq s<e-1=|Q \cap W|$. If $s=e-1$ and $2 e+1 \in P$, then $W=(P \cap Z) \cup\{2 e+1\} \in \mathcal{M}$ and $|P \cap W|=e>e-1=|Q \cap W|$. If $s=e-1$ and $2 e+1 \notin P$, let $W_{1}$ be an $(e-1)$-subset of $Z$ satisfying $\left|P \cap W_{1}\right|=s-1$. Then $W=W_{1} \cup\{2 e+1\} \in \mathcal{M}$ and $|P \cap W|=s-1<e-1=|Q \cap W|$.

Case 1.2: $r=1$. If $s=0$ and either $2 e+1 \in Q$ or $2 e+1 \notin P \cup Q$, let $W_{1}$ be an $(e-1)$-subset of $Z$ containing $Q \cap Z$. Then $W=W_{1} \cup\{2 e+1\} \in \mathcal{M}$ and $|P \cap W|<|Q \cap W|$. If $s=0,2 e+1 \in P$ and $2 e+1 \notin Q$, take $W_{1}=Z \backslash(Q \cap Z)$. Then $W=W_{1} \cup\{2 e+1\} \in \mathcal{M}$ and $|P \cap W|=1>0=|Q \cap W|$. If $s=1$ and $2 e+1 \in P$, let $W_{1}=Z \backslash(Q \cap Z)$. Then $W=W_{1} \cup\{2 e+1\} \in \mathcal{M}$ and $|P \cap W|=2>1 \geq|Q \cap W|$. If $s=1$ and $2 e+1 \notin P$, let $W_{1}=Z \backslash(P \cap Z)$. Then $W=W_{1} \cup\{2 e+1\} \in \mathcal{M}$ and $|P \cap W|=0<1 \leq|Q \cap W|$.

Case 1.3: $1<r<e$. If $0 \leq s \leq r-2$, take an $(e-1)$-subset $W_{1}$ of $Z$ containing $Q \cap Z$. Then $W=W_{1} \cup\{2 e+1\} \in \mathcal{M}$ and $|P \cap W| \leq s+1<r \leq|Q \cap W|$. If $s=r-1$ and either $2 e+1 \in Q$ or $2 e+1 \notin P \cup Q$, let $W_{1}$ be an $(e-1)$-subset of $Z$ containing $Q \cap Z$. Then $W=W_{1} \cup\{2 e+1\} \in \mathcal{M}$ and $|P \cap W|<|Q \cap W|$. If $s=r-1,2 e+1 \in P$ and $2 e+1 \notin Q$, let $W_{1}$ be an $(e-1)$-subset of $Z$ satisfying $P \cap Z \subseteq W_{1}$ and $\left|W_{1} \cap Q \cap Z\right|=r-1$. Then $W=W_{1} \cup\{2 e+1\} \in \mathcal{M}$ and $|P \cap W|=s+1>r-1=|Q \cap W|$. If $s=r$ and $2 e+1 \in Q$, let $Q \cap Z=\left\{\alpha_{1}, \ldots, \alpha_{r}\right\}$. Since $P \cap Z \neq Q \cap Z$, there exists a $\beta \in(P \cap Z) \backslash(Q \cap Z)$ such that $\left\{\alpha_{1}, \ldots, \alpha_{r}, \beta\right\}$ is an $(r+1)$-subset. Let $Z=\left\{\alpha_{1}, \ldots, \alpha_{r}, \beta, \gamma_{1}, \ldots, \gamma_{e-r-1}\right\}$ and $W_{1}=\left\{\alpha_{1}, \ldots, \alpha_{r}, \gamma_{1}, \ldots, \gamma_{e-r-1}\right\}$. Then $W=W_{1} \cup\{2 e+1\} \in \mathcal{M}$. By $\beta \notin W$ and $\beta \in P \cap Z,|P \cap W| \leq|P \cap Z|=$ $r<r+1=|Q \cap W|$. If $s=r$ and $2 e+1 \in P$, then there exists a $W \in \mathcal{M}$ such that $|P \cap W|=r+1>r \geq|Q \cap W|$. If $s=r$ and $2 e+1 \notin P \cup Q$, then there exists an $(e-1)$-subset $W_{1}$ such that $\left|P \cap W_{1}\right|<\left|Q \cap W_{1}\right|$. It follows that $W=W_{1} \cup\{2 e+1\} \in \mathcal{M}$ and $|P \cap W|<|Q \cap W|$.

Case 2: $P \cap[2 e]=Q \cap[2 e]$. Then $P \cap[e]=Q \cap[e]$. Let $W_{1}$ be an $(e-1)$-subset of $[e]$. Then $W=W_{1} \cup\{2 e+1\} \in \mathcal{M}$ and $|P \cap W| \neq|Q \cap W|$.

Hence, $\mathcal{M}$ is a resolving set. Finally, we obtain the bound by observing that $|\mathcal{M}|=2 e$.

\section{Doubled Odd graphs and their $q$-analogue}

The doubled Odd graph, denoted by $O(2 e+1, e, e+1)$, is a bipartite graph with bipartition $\left(\begin{array}{c}{[2 e+1]} \\ e\end{array}\right) \cup\left(\begin{array}{c}{[2 e+1]} \\ e+1\end{array}\right)$ such that two vertices $P \in\left(\begin{array}{c}{[2 e+1]} \\ e\end{array}\right)$ and $Q \in\left(\begin{array}{c}{[2 e+1]} \\ e+1\end{array}\right)$ are adjacent if and only if $P \subseteq Q$. Then $O(2 e+1, e, e+1)$ is a distance-regular graph with diameter $2 e+1$ (see [6]). For any two vertices $P$ and $Q$, we have $d(P, Q)=2 i+|| P|-| Q||$ if and only if $|P \cap Q|=\min \{|P|,|Q|\}-i$. For $e=1, O(3,1,2)$ is a hexagon. It is obvious that $\mu(O(3,1,2))=2 e$. For $e \geq 2$, we obtain the following result.

Theorem 3.1. Let $e \geq 2$. Then $\mu(O(2 e+1, e, e+1)) \leq 2 e+1$.

Proof. We give an explicit construction of a resolving set.

Let $Y=[2 e+1] \backslash[e+1], \mathcal{W}=\{W \subseteq[e+1]|| W \mid=e\}$ and $\mathcal{Y}=\left\{W \cup\{1\} \mid W \in\left(\begin{array}{c}Y \\ e-1\end{array}\right)\right\}$. We will show that $\mathcal{M}=\mathcal{W} \cup \mathcal{Y}$ is a resolving set. Since $O(2 e+1, e, e+1)$ is bipartite, we only need to show that, for any two distinct vertices $P$ and $Q$ in the same part, there exists a vertex $W \in \mathcal{M}$ such that $|P \cap W| \neq|Q \cap W|$. 
Suppose $P, Q \in\left(\begin{array}{c}{[2 e+1]} \\ e+i\end{array}\right)$, where $i=0$ or 1 .

Case 1: $P \cap[e+1] \neq Q \cap[e+1]$. Suppose $s+i=|P \cap[e+1]| \leq|Q \cap[e+1]|=r+i$. Then $1-i \leq r \leq e$.

Case 1.1: $s<r$. For $i=0$, let $W$ be an $e$-subset of $[e+1]$ containing $Q \cap[e+1]$. Then $W \in \mathcal{M}$ and

$$
|P \cap W| \leq|P \cap[e+1]|=s<r=|Q \cap W| .
$$

For $i=1$, let $W$ be an $e$-subset of [ $e+1]$ satisfying $|P \cap W|=s$. Then $W \in \mathcal{M}$ and $|P \cap W|=s<r \leq|Q \cap W|$.

Case 1.2: $s=r$. Let $Q \cap[e+1]=\left\{\alpha_{1}, \ldots, \alpha_{r}, \alpha_{r+i}\right\}$. Since $P \cap[e+1] \neq Q \cap[e+1]$, there exists a $\beta \in(P \cap[e+1]) \backslash(Q \cap$ $[e+1])$ such that $\left\{\alpha_{1}, \ldots, \alpha_{r}, \alpha_{r+i}, \beta\right\}$ is an $(r+1+i)$-subset of $[e+1]$. Let $[e+1]=\left\{\alpha_{1}, \ldots, \alpha_{r}, \alpha_{r+i}, \beta, \gamma_{1}, \ldots, \gamma_{e-r-i}\right\}$ and $W=\left\{\alpha_{1}, \ldots, \alpha_{r}, \alpha_{r+i}, \gamma_{1}, \ldots, \gamma_{e-r-i}\right\}$. Then $W \in \mathcal{M}$. By $\beta \notin W$ and $\beta \in P \cap[e+1]$,

$$
|P \cap W|<|P \cap[e+1]|=|Q \cap[e+1]|=r+i=|Q \cap W| .
$$

Case 2: $P \cap[e+1]=Q \cap[e+1]$. Then $P \cap Y \neq Q \cap Y$ and $|P \cap Y|=|Q \cap Y|$. Suppose $|P \cap Y|=|Q \cap Y|=r$. Then $0<r<e$. Let $Q \cap Y=\left\{\alpha_{1}, \ldots, \alpha_{r}\right\}$. Since $P \cap Y \neq Q \cap Y$, there exists a $\beta \in(P \cap Y) \backslash(Q \cap Y)$ such that $\left\{\alpha_{1}, \ldots, \alpha_{r}, \beta\right\}$ is an $(r+1)$-subset of $Y$. Let $Y=\left\{\alpha_{1}, \ldots, \alpha_{r}, \beta, \gamma_{1}, \ldots, \gamma_{e-r-1}\right\}$ and $W_{1}=\left\{\alpha_{1}, \ldots, \alpha_{r}, \gamma_{1}, \ldots, \gamma_{e-r-1}\right\}$. By $\beta \notin W_{1}$ and $\beta \in P \cap Y$,

$$
\left|P \cap W_{1}\right|<|P \cap Y|=|Q \cap Y|=r=\left|Q \cap W_{1}\right| .
$$

Therefore, $W=W_{1} \cup\{1\} \in \mathcal{M}$ and $|P \cap W| \neq|Q \cap W|$.

Hence, $\mathcal{M}$ is a resolving set. Finally, we obtain the bound by observing that $|\mathcal{M}|=2 e+1$.

Let $\mathbb{F}_{q}$ be a finite field with $q$ elements, where $q$ is a prime power. For a non-negative integer $n$, let $\mathbb{F}_{q}^{n}$ be an $n$ dimensional vector space over $\mathbb{F}_{q}$. For a non-negative integer $m \leq n$, let $\left[\begin{array}{c}{[n]} \\ m\end{array}\right]$ be the set of all $m$-dimensional subspaces of $\mathbb{F}_{q}^{n}$. Then the size of $\left[\begin{array}{c}{[n]} \\ m\end{array}\right]$ is $\left[\begin{array}{c}n \\ m\end{array}\right]_{q}=\prod_{i=n-m+1}^{n}\left(q^{i}-1\right) / \prod_{i=1}^{m}\left(q^{i}-1\right)$.

A partition of the vector space $V$ is a set $\mathcal{P}$ of subspaces of $V$ such that any non-zero vector is contained in exactly one element of $\mathcal{P}$. If $T=\{\operatorname{dim} W \mid W \in \mathcal{P}\}$, the partition $\mathcal{P}$ is said to be a $T$-partition of $V$.

Let $n=2 e+1$ and $e \geq 1$. The doubled Grassmann graph, denoted by $J_{q}(2 e+1, e, e+1)$, is a bipartite graph with bipartition $\left[\begin{array}{c}{[2 e+1]} \\ e\end{array}\right] \cup\left[\begin{array}{c}{[2 e+1]} \\ e+1\end{array}\right]$ such that two vertices $P \in\left[\begin{array}{c}{[2 e+1]} \\ e\end{array}\right]$ and $Q \in\left[\begin{array}{c}{[2 e+1]} \\ e+1\end{array}\right]$ are adjacent if and only if $P \subseteq Q$. Then $J_{q}(2 e+1, e, e+1)$ is a distance-regular graph with diameter $2 e+1$ (see [6]). For any two vertices $P$ and $Q$, we have $d(P, Q)=2 i+|\operatorname{dim} P-\operatorname{dim} Q|$ if and only if $\operatorname{dim}(P \cap Q)=\min \{\operatorname{dim} P, \operatorname{dim} Q\}-i$. For $e=1$, it is obvious that $\mu\left(J_{q}(3,1,2)\right)=q(q+1)$. For $e \geq 2$, we obtain the following result.

Theorem 3.2. Let $e \geq 2$. Then $\mu\left(J_{q}(2 e+1, e, e+1)\right) \leq\left(q^{2 e+2}-1\right) /(q-1)$.

Proof. By [5, Lemma 3], there exists an $\{e+1, e\}$-partition $\mathcal{P}=\left\{X, Y_{1}, \ldots, Y_{q^{e+1}}\right\}$ of $\mathbb{F}_{q}^{2 e+1}$, where $\operatorname{dim} X=e+1$ and $\operatorname{dim} Y_{i}=e, i=1,2, \ldots, q^{e+1}$. For a fixed 1-dimensional subspace $U$ of $X$, let

$$
\mathcal{W}=\left\{W \subseteq U \oplus Y \mid \operatorname{dim} W=e, Y \in\left\{Y_{1}, \ldots, Y_{q^{e+1}}\right\}\right\}, \quad X=\{W \subseteq X \mid \operatorname{dim} W=e\} .
$$

To prove $\mathcal{M}=\mathcal{W} \cup \mathcal{X}$ is a resolving set, we only need to show that, for any two distinct vertices $P$ and $Q$ in the same part, there exists a vertex $W \in \mathcal{M}$ such that $\operatorname{dim}(P \cap W) \neq \operatorname{dim}(Q \cap W)$.

Suppose $P, Q \in\left[\begin{array}{c}{[2 e+1]} \\ e+i\end{array}\right]$, where $i=0$ or 1 .

Case 1: $P \cap X \neq Q \cap X$. Suppose $s+i=\operatorname{dim}(P \cap X) \leq \operatorname{dim}(Q \cap X)=r+i$. Then $1-i \leq r \leq e$.

Case 1.1: $s<r$. For $i=0$, let $W$ be an $e$-dimensional subspace of $X$ containing $Q \cap X$. Then $W \in \mathcal{M}$ and

$$
\operatorname{dim}(P \cap W) \leq s<r=\operatorname{dim}(Q \cap W) .
$$

For $i=1$, let $W$ be an $e$-dimensional subspace of $X$ satisfying $\operatorname{dim}(P \cap W)=s$. Then $W \in \mathcal{M}$ and $\operatorname{dim}(P \cap W)=s<$ $e=\operatorname{dim}(Q \cap W)$.

Case 1.2: $s=r$. Let $\left\{\alpha_{1}, \ldots, \alpha_{r}, \alpha_{r+i}\right\}$ be a basis for $Q \cap X$. Since $P \cap X \neq Q \cap X$, there exists a $\beta \in(P \cap X) \backslash(Q \cap X)$ such that $\left\{\alpha_{1}, \ldots, \alpha_{r}, \alpha_{r+i}, \beta\right\}$ is linearly independent. Extend this to a basis $\left\{\alpha_{1}, \ldots, \alpha_{r}, \alpha_{r+i}, \beta, \gamma_{1}, \ldots, \gamma_{e-r-i}\right\}$ for $X$. 
Let $W$ be the $e$-dimensional subspace spanned by $\left\{\alpha_{1}, \ldots, \alpha_{r}, \alpha_{r+i}, \gamma_{1}, \ldots, \gamma_{e-r-i}\right\}$. Then $W \in \mathcal{M}$. By $\beta \notin W$ and $\beta \in P \cap X$

$$
\operatorname{dim}(P \cap W)<\operatorname{dim}(P \cap X)=\operatorname{dim}(Q \cap X)=r+i=\operatorname{dim}(Q \cap W) .
$$

Case 2: $P \cap X=Q \cap X$. Then there exists a $Y \in\left\{Y_{1}, \ldots, Y_{q^{e+1}}\right\}$ such that $P \cap Y \neq Q \cap Y$. Then $P \cap(U \oplus Y) \neq Q \cap(U \oplus Y)$. Similar to the proof of Case 1 , there exists a $W \in \mathcal{M}$ such that $\operatorname{dim}(P \cap W) \neq \operatorname{dim}(Q \cap W)$.

Hence, $\mathcal{M}$ is a resolving set. Finally, we obtain the bound by observing that

$$
|\mathcal{M}|=\left[\begin{array}{c}
e+1 \\
1
\end{array}\right]_{q}+q^{e+1}\left[\begin{array}{c}
e+1 \\
1
\end{array}\right]_{q}=\frac{q^{2 e+2}-1}{q-1} .
$$

\section{Twisted Grassmann graphs}

For $e \geq 2$, let $H$ be a fixed $2 e$-dimensional subspace of $\mathbb{F}_{q}^{2 e+1}$,

$$
\begin{aligned}
& \mathcal{B}_{1}:=\left\{W \text { a subspace of } \mathbb{F}_{q}^{2 e+1} \mid \operatorname{dim} W=e+1, W \nsubseteq H\right\}, \\
& \mathcal{B}_{2}:=\quad\left\{W \text { a subspace of } \mathbb{F}_{q}^{2 e+1} \mid \operatorname{dim} W=e-1, W \subseteq H\right\} .
\end{aligned}
$$

The twisted Grassmann graph $\tilde{J}_{q}(2 e+1, e)$ has the vertex set $\mathcal{B}_{1} \cup \mathcal{B}_{2}$, and two vertices $P$ and $Q$ are adjacent if and only if $\operatorname{dim} P+\operatorname{dim} Q-2 \operatorname{dim}(P \cap Q)=2$. Dam and Koolen [10] constructed the graph, and showed that $\tilde{J}_{q}(2 e+1, e)$ is a distance-regular graph, which is the first know family of non-vertex-transitive distance-regular graphs with unbounded diameter. For any two vertices $P$ and $Q, d(P, Q)=i$ if and only if $\operatorname{dim} P+\operatorname{dim} Q-2 \operatorname{dim}(P \cap Q)=2 i$.

Theorem 4.1. Let $e \geq 2$. Then $\mu\left(\tilde{J}_{q}(2 e+1, e)\right) \leq\left(q^{2 e}\left(q^{e+2}-q+1\right)-1\right) /(q-1)$.

Proof. For $e \geq 2$, by [5, Lemma 3], there exists an $\{e, 1\}$-partition $\mathcal{P}=\left\{X_{1}, \ldots, X_{q^{e}+1}, Y_{1}, \ldots, Y_{q^{2 e}}\right\}$ of $\mathbb{F}_{q}^{2 e+1}$, where $\left\{X_{1}, \ldots, X_{q^{e}+1}\right\}$ is an $\{e\}$-partition of $H$ and $\operatorname{dim} Y_{i}=1, i=1, \ldots, q^{2 e}$. For a fixed $(e+1)$-dimensional subspace $U$ of $H$, let

$\mathcal{W}=\left\{W \subseteq U \oplus Y \mid \operatorname{dim} W=e+1, W \neq U, Y \in\left\{Y_{1}, \ldots, Y_{q^{2 e}}\right\}\right\}, X=\left\{W \subseteq X \mid \operatorname{dim} W=e-1, X \in\left\{X_{1}, \ldots, X_{q^{e}+1}\right\}\right\}$.

To prove $\mathcal{M}=\mathcal{W} \cup \mathcal{X}$ is a resolving set, it is sufficient to show that, for any two distinct vertices $P$ and $Q$, there exists a vertex $W \in \mathcal{M}$ such that

$$
\begin{cases}\operatorname{dim}(P \cap W) \neq \operatorname{dim}(Q \cap W), & \text { if } P, Q \in \mathcal{B}_{i} ; \\ \operatorname{dim}(P \cap W)-\operatorname{dim}(Q \cap W) \neq 1, & \text { if } P \in \mathcal{B}_{1}, Q \in \mathcal{B}_{2} .\end{cases}
$$

Firstly, we assume that $P, Q \in \mathcal{B}_{2}$. Similar to the proof of [3, Proposition 7], there exists a $W \in X$ such that $\operatorname{dim}(P \cap W) \neq \operatorname{dim}(Q \cap W)$.

Secondly, we assume that $P, Q \in \mathcal{B}_{1}$.

Case 1: $P \cap H \neq Q \cap H$. Since $\left\{X_{1}, \ldots, X_{q^{e}+1}\right\}$ is a partition of $H$, there exists an $X \in\left\{X_{1}, \ldots, X_{q^{e}+1}\right\}$ such that $P \cap X \neq Q \cap X$. Suppose $s=\operatorname{dim}(P \cap X) \leq \operatorname{dim}(Q \cap X)=r$. Then $0<r \leq e$.

Case 1.1: $r=e$. Then $X \subseteq Q$. Since $\operatorname{dim}(Q \cap H)=e, Q \cap X=Q \cap H$. Since $P \cap X \neq Q \cap X$, $\operatorname{dim}(P \cap X)<e$. If $s=0$, take an $(e-1)$-dimensional $W$ of $X$. Then $W \in \mathcal{M}$ and $0=\operatorname{dim}(P \cap W)<e-1=\operatorname{dim}(Q \cap W)$. If $s \geq 1$, let $W$ be an $(e-1)$-dimensional subspace of $X$ satisfying $\operatorname{dim}(P \cap W)=s-1$. Then $W \in \mathcal{M}$ and

$$
\operatorname{dim}(P \cap W)=s-1<e-1=\operatorname{dim}(Q \cap W)
$$

Case 1.2: $s<r<e$. Let $W$ be an $(e-1)$-dimensional subspace of $X$ containing $Q \cap X$. Then $W \in \mathcal{M}$ and

$$
\operatorname{dim}(P \cap W) \leq s<r=\operatorname{dim}(Q \cap W) .
$$


Case 1.3: $s=r<e$. Let $\left\{\alpha_{1}, \ldots, \alpha_{r}\right\}$ be a basis for $Q \cap X$. Since $P \cap X \neq Q \cap X$, there exists a $\beta \in(P \cap X) \backslash(Q \cap X)$ such that $\left\{\alpha_{1}, \ldots, \alpha_{r}, \beta\right\}$ is linearly independent. Extend this to a basis $\left\{\alpha_{1}, \ldots, \alpha_{r}, \beta, \gamma_{1}, \ldots, \gamma_{e-r-1}\right\}$ for $X$. Let $W$ be the $(e-1)$-dimensional subspace spanned by $\left\{\alpha_{1}, \ldots, \alpha_{r}, \gamma_{1}, \ldots, \gamma_{e-r-1}\right\}$. Then $W \in \mathcal{M}$. By $\beta \notin W$ and $\beta \in P \cap X$,

$$
\operatorname{dim}(P \cap W)<\operatorname{dim}(P \cap X)=\operatorname{dim}(Q \cap X)=r=\operatorname{dim}(Q \cap W) .
$$

Case 2: $P \cap H=Q \cap H$. Then there exists a $Y \in\left\{Y_{1}, \ldots, Y_{q^{2 e}}\right\}$ such that $P \cap Y \neq Q \cap Y$. Then $P \cap(U \oplus Y) \neq Q \cap(U \oplus Y)$. Suppose $s=\operatorname{dim}(P \cap(U \oplus Y)) \leq \operatorname{dim}(Q \cap(U \oplus Y))=r$. Then $2 \leq s$ and $r \leq e+1$.

Case 2.1: $r=e+1$. Then $Q \subseteq U \oplus Y$ but $Q \neq U$, which follows that $Q \in \mathcal{M}$ and $\operatorname{dim}(P \cap Q)<e+1$.

Case 2.2: $s<r<e+1$. Then exists an $(e+1)$-dimensional subspace $W$ of $U \oplus Y$ such that $\operatorname{dim}(P \cap W)=s-1<$ $r-1 \leq \operatorname{dim}(Q \cap W)$. Since $P \cap U=Q \cap U, W \neq U$, which implies that $W \in \mathcal{M}$.

Case 2.3: $s=r<e+1$. Then $Q \cap(U \oplus Y) \neq Q \cap U$. Indeed, if $Q \cap(U \oplus Y)=Q \cap U, P \cap U=Q \cap U$ by $U \subseteq H$. Since $P \cap U \subseteq P \cap(U \oplus Y)$ and $\operatorname{dim}(P \cap U) \leq s \leq r=\operatorname{dim}(Q \cap U), P \cap U=P \cap(U \oplus Y)$, a contradiction. Let $\left\{\alpha_{1}, \ldots, \alpha_{r}\right\}$ be a basis for $Q \cap(U \oplus Y)$. Since $P \cap(U \oplus Y) \neq Q \cap(U \oplus Y)$, there exists a $\beta \in(P \cap(U \oplus Y)) \backslash(Q \cap(U \oplus Y))$ such that $\left\{\alpha_{1}, \ldots, \alpha_{r}, \beta\right\}$ is linearly independent. Extend this to a basis $\left\{\alpha_{1}, \ldots, \alpha_{r}, \beta, \gamma_{1}, \ldots, \gamma_{e+1-r}\right\}$ for $U \oplus Y$. Let $W$ be the $(e+1)$-dimensional subspace spanned by $\left\{\alpha_{1}, \ldots, \alpha_{r}, \gamma_{1}, \ldots, \gamma_{e+1-r}\right\}$. Then $W \neq U$ by $Q \cap(U \oplus Y) \neq Q \cap U$, which implies that $W \in \mathcal{M}$. By $\beta \notin W$ and $\beta \in P \cap(U \oplus Y)$,

$$
\operatorname{dim}(P \cap W)<\operatorname{dim}(P \cap(U \oplus Y))=\operatorname{dim}(Q \cap(U \oplus Y))=r=\operatorname{dim}(Q \cap W) .
$$

Next, we assume that $P \in \mathcal{B}_{1}, Q \in \mathcal{B}_{2}$.

Case 3.1: $Q \in \mathcal{M}$. Then $\operatorname{dim}(P \cap Q)-\operatorname{dim} Q \neq 1$.

Case 3.2: $Q \notin \mathcal{M}$. Since $\left\{X_{1}, \ldots, X_{q^{e}+1}\right\}$ is a partition of $H$ and $Q \subseteq H$, there exists an $X \in\left\{X_{1}, \ldots, X_{q^{e}+1}\right\}$ such that $e-2 \geq s=\operatorname{dim}(Q \cap X) \geq 1$. Let $\operatorname{dim}(P \cap X)=r$. Then $0 \leq r \leq e$.

Case 3.2.1: $r>s+1$. If $r=e$, then $X \subseteq P$. Let $\left\{\alpha_{1}, \ldots, \alpha_{s}\right\}$ be a basis for $Q \cap X$. Extend this to a basis $\left\{\alpha_{1}, \ldots, \alpha_{s}, \gamma_{1}, \ldots, \gamma_{e-s}\right\}$ for $X$. Let $W$ be the $(e-1)$-dimensional subspace spanned by $\left\{\alpha_{2}, \ldots, \alpha_{s}, \gamma_{1}, \ldots, \gamma_{e-s}\right\}$. Then $W \in \mathcal{M}$. By $\alpha_{1} \notin W, \operatorname{dim}(Q \cap W)=s-1$. It follows that $\operatorname{dim}(P \cap W)-\operatorname{dim}(Q \cap W)=e-s>1$. Now let $r<e$. Suppose $W$ is an $(e-1)$-dimensional subspace of $X$ containing $P \cap X$. Then $W \in \mathcal{M}$ and $\operatorname{dim}(Q \cap W) \leq s$. It follows that $\operatorname{dim}(P \cap W)-\operatorname{dim}(Q \cap W) \geq r-s>1$.

Case 3.2.2: $r \leq s$. Let $W$ be an $(e-1)$-dimensional subspace of $X$ containing $Q \cap X$. Then $W \in \mathcal{M}, Q \cap X=Q \cap W$ and $\operatorname{dim}(P \cap W) \leq r$. It follows that $\operatorname{dim}(P \cap W)-\operatorname{dim}(Q \cap W) \leq r-s<1$.

Case 3.2.3: $r=s+1$. If $Q \cap X \subseteq P \cap X$, then let $\left\{\alpha_{1}, \ldots, \alpha_{s}, \alpha_{s+1}\right\}$ be a basis for $P \cap X$, where $\left\{\alpha_{1}, \ldots, \alpha_{s}\right\}$ is a basis for $Q \cap X$. Extend this to a basis $\left\{\alpha_{1}, \ldots, \alpha_{s+1}, \gamma_{1}, \ldots, \gamma_{e-s-1}\right\}$ for $X$. Let $W$ be the $(e-1)$-dimensional subspace spanned by $\left\{\alpha_{1}, \ldots, \alpha_{s}, \gamma_{1}, \ldots, \gamma_{e-s-1}\right\}$. Then $W \in \mathcal{M}$. By $\alpha_{s+1} \notin W, \operatorname{dim}(P \cap W)=s$, which implies that $\operatorname{dim}(P \cap W)-\operatorname{dim}(Q \cap W)=0$. If $Q \cap X \nsubseteq P \cap X$, pick a basis $\left\{\alpha_{1}, \alpha_{2}, \ldots, \alpha_{s}\right\}$ for $Q \cap X$. Then there exists a $\beta \in(P \cap X) \backslash(Q \cap X)$ such that $\left\{\alpha_{1}, \ldots, \alpha_{s}, \beta\right\}$ is linearly independent. Extend this to a basis $\left\{\alpha_{1}, \ldots, \alpha_{s}, \beta, \gamma_{1}, \ldots, \gamma_{e-s-1}\right\}$ for $X$. Let $W$ be the $(e-1)$-dimensional subspace spanned by $\left\{\alpha_{1}, \ldots, \alpha_{s}, \gamma_{1}, \ldots, \gamma_{e-s-1}\right\}$. Then $W \in \mathcal{M}$. By $\beta \notin W$ and $\beta \in P \cap X, \beta \notin P \cap W$. Hence $\operatorname{dim}(P \cap W) \leq s$ and $\operatorname{dim}(P \cap W)-\operatorname{dim}(Q \cap W) \leq 0$.

Hence, $\mathcal{M}$ is a resolving set. Finally, we obtain the bound by observing that

$$
|\mathcal{M}|=\left(q^{e}+1\right)\left[\begin{array}{l}
e \\
1
\end{array}\right]_{q}+q^{2 e}\left(\left[\begin{array}{c}
e+2 \\
1
\end{array}\right]_{q}-1\right)=\frac{q^{2 e}\left(q^{e+2}-q+1\right)-1}{q-1} .
$$

Babai [1] obtained bounds on a parameter of primitive distance-regular graphs which is equivalent to the metric dimension. A natural question is to compare our result with those. For the case of the twisted Grassmann graph $\tilde{J}_{q}(2 e+1, e)$, Babai's most general bound (see [1, Theorem 2.1]) yields

$$
\mu\left(\tilde{J}_{q}(2 e+1, e)\right)<4 \sqrt{\left[\begin{array}{c}
2 e+1 \\
e
\end{array}\right]_{q}} \log \left[\begin{array}{c}
2 e+1 \\
e
\end{array}\right]_{q}
$$

while his stronger bound (see [1, Theorem 2.4]) yields

$$
\mu\left(\tilde{J}_{q}(2 e+1, e)\right)<2 e \frac{\left[\begin{array}{c}
2 e+1 \\
e
\end{array}\right]_{q}}{\left[\begin{array}{c}
2 e+1 \\
e
\end{array}\right]_{q}-M} \log \left[\begin{array}{c}
2 e+1 \\
e
\end{array}\right]_{q},
$$


where

$$
M=\max _{1 \leq j \leq e} q^{j^{2}}\left[\begin{array}{c}
e+1 \\
j
\end{array}\right]_{q}\left[\begin{array}{l}
e \\
j
\end{array}\right]_{q} .
$$

These bounds are difficult to evaluate exactly, so we conducted some experiments using MATLAB to compare these bounds with the one obtained in Theorem 3.2. Our experiments indicate that our constructive bound is an improvement on Babai's general bounds in most of cases, but Babai's bound seems better than our bound for small $q$.

\section{Acknowledgement}

This research is partially supported by NSF of China (10971052, 10871027), NCET-08-0052, Langfang Teachers' College (LSZB201005), Hunan Provincial Natural Science Foundation of China (09JJ3006), and the Fundamental Research Funds for the Central Universities of China.

\section{References}

[1] L. Babai, On the order of uniprimitive permutation groups, Ann. Math. 113 (1981) 553-568.

[2] R.F. Bailey, J. Cáceres, D. Garijo, A. González, A. Márquez, K. Meagher and M.L. Puertas, Resolving sets in Johnson and Kneser graphs, Preprint.

[3] R.F. Bailey and K. Meagher, On the metric dimension of Grassmann graphs, arXiv:1010.4495 2 [math.CO].

[4] R.F. Bailey and P.J. Cameron, Base size, metric dimension an other invariants of groups and graphs, Bull. London Math. Soc. 43 (2011) 209-242.

[5] A. Beutelspacher, Partition of finite vector spaces: an application of Frobenius number in geometry, Arch. Math. (Basel) 31 (1978) $202-208$.

[6] A.E. Brouwer, A.M. Cohen and A. Neumaier, Distance-Regular Graphs, Springer-Verlag, Berlin, 1989.

[7] J. Cáceres, C. Hernando, M. Mora, I.M. Pelayo, M.L. Puertas, C. Seara and D.R. Wood, On the metric dimension of Cartesian products of graphs, SIAM J. Discrete Math. 21 (2007) 423-441.

[8] G. Chartrand, L. Eroh, M.A. Johnson and O.R. Oellermann, Resolvability in graphs and the metric dimension of a graph, Discrete Appl. Math. 105 (2000) 99-113.

[9] V. Chvátal, Mastermind, Combinatorica 3 (1983) 325-329.

[10] E.R. van Dam and J.H. Koolen, A new family of distance-regular graphs with unbounded diameter, Inventiones Mathematicae 162 (2005) 189-193.

[11] M. Feng and K. Wang, On the metric dimension of bilinear forms graphs, arXiv:1104.4089 1 [math.CO].

[12] F. Harary and R.A. Melter, On the metric dimension of a graph, Ars Combin. 2(1976) 191-195; 4 (1977) 318.

[13] P.J. Slater, Leaves of trees, Conger. Number. 14 (1975) 549-559. 